\title{
On some generalisations of Laguerre polynomials
}

\author{
By A. Erdélyi.
}

(Received 16th January, 1940. Read 3rd February, 1940.)

\section{Introduction.}

1. The sequence of orthogonal functions derived from Laguerrepolynomials is known to be complete, and hence closed, in $L^{2}\left(0, \infty_{\text {s }}\right)$ if $\mathscr{R}(a)>-1$. In a recent paper $^{1}$ Dr Kober introduced a generalisation of this sequence, which enables him to extend the known results also for $\mathcal{R}(a)<-1$. Kober's guiding principle seems to be the following one: The Laguerre orthogonal functions form, for $\mathscr{R}(a)>-1$, a complete system of self and skew reciprocal functions of the Hankel transformation of order $a$. Now, if $\mathcal{Q}(a)<-1$, the ordinary Hankel transform has to be replaced by the so-called cut Hankel transform. Hence the system of functions which has to replace. Laguerre orthogonal functions when $\mathscr{R}(a)<-1$, should be a com. plete system of self and skew reciprocal functions of the cut Hankel transformation of order $\alpha$, such that it reduces for $\mathcal{R}(a)>-1$ (when the cut Hankel transform reduces to the ordinary one) to the sequence of Laguerre orthogonal functions. This, of course, is by no means a unique definition; nevertheless, together with what one would call the permanence of the Mellin transform, it enabled Kober to find a sequence of functions $\left\{\psi_{n, m}^{(a)}\right\}$ which (i) reduces to the sequence of Laguerre orthogonal functions when $\mathscr{R}(a)>-1, m=0$, (ii) is a. complete set of self and skew reciprocal functions of the cut Hankel transformation with kernel $J_{a, m}$ and (iii) has the required qualities of completeness and closedness.

When reading Kober's paper, I realised that, at least formally, the cut Hankel transform with kernel $J_{a_{r} m}$ can be derived from the ordinary Hankel transform of order $a+2 m$ by partial integration. Thus it should be possible to derive a system of self and skew reciprocal functions of the kernel $J_{a, m}$ directly from the eigen-functions of the kernel $J_{a+2 m}$, i.e. from Laguerre orthogonal functions of index $a+2 m$. I wondered if this process would yield Kober's functions:

${ }^{1}$ H. Kolser, Proc. Edinburgh Math. Soc. (2), 6 (1940), 135-146. 
$\psi_{n, m}^{(a)}$, and if not, whether the system of reciprocal functions obtained thus is an orthogonal one (Kober's functions $\psi_{n, m}^{(a)}$ are not orthogonal to each other). Actually the reduction by partial integration can be performed in two different ways. Correspondingly I obtained two different sets of eigen-functions of the cut Hankel transformation, which will be denoted in the present paper by $\left\{\chi_{n}^{(a, m)}\right\}$ and $\left\{\phi_{n}^{(\alpha . m)}\right\}$ respectively $(n=0,1,2, \ldots)$. Neither of these two sequences is identical with Kober's $\left\{\psi_{n, m}^{(a)}\right\}$; neither of them is an orthogonal set. But in a way they seem to be the simplest systems of reciprocal functions of the cut Hankel transform, and their principal feature is, that taken together they form a biorthogonal system $\left\{\chi_{n}^{(a, m)}, \phi_{n}^{(a, m)}\right\}$.

The sequence $\left\{\phi_{n}^{(\alpha, m)}\right\}$ is especially worth mentioning. Like Laguerre's orthogonal set $\left\{\psi_{n}^{(a)}\right\} \equiv\left\{\phi_{n}^{(a)}\right\} \equiv\left\{\chi_{n}^{(a)}\right\},(\mathscr{R}(a)>-1, m=0)$, it consists, even in the general case $m \neq 0$, of functions which, except for a common factor, are polynomials. These polynomials can be expressed by certain polynomials of two variables which I introduced a few years ago.

In the second section of the present paper I recall those of the known properties of the Hankel transform $[\mathcal{R}(\alpha)>-1]$ which are required and/or generalised in what follows. In section 3 the connection between the ordinary and cut Hankel transform is stated only; the proof of the general theorem has been published elsewhere. For the purposes of the present paper it is sufficient to give the application of this theorem to the special sets of reciprocal functions dealt with in this paper. That the functions defined thus are really reciprocal in the cut Hankel transform, is readily seen from their Mellin transforms, given in sections 7 and 9 respectively.

In sections 4-6 the formal properties of the polynomials and of some slightly generalised polynomials are investigated. For the sake of brevity only those formulae are given which are required in subsequent work. Section 8 contains the corresponding formulae for certain functions $\Lambda$ which are connected in a similar way with $\left\{\phi_{n}^{(a, m)}\right\}$. In sections 10 and 11 the biorthogonality and completeness of the system $\left\{\chi_{n}^{(a . m)}, \phi_{n}^{(a . m)}\right\}$ is proved.

The following sections contain a rough sketch of alternative proofs of some of the results obtained in the first part of the paper. This sketch is meant to illustrate at the same time some points of the theory of general transforms. A more exact statement of the results of this part, as regards the theory of general transforms, and a rigorous 
proof $I$ hope to give in a future paper. In the twelfth section it is pointed out that from any reciprocal function of a general transform an infinity of sequences of reciprocal functions can be generated. The orthogonality, biorthogonality, completeness and closedness of these sequences can be investigated by the help of the generating function (section 13). In sections 14 and 15, I point out that from the generating function an integral operator can be derived which is of some importance for the transform dealt with.

Hitherto I followed Kober in discussing such generalisations of Laguerre's orthogonal set which consist of reciprocal functions of cut Hankel transforms. So the developments of the main part of the paper may also be of some interest from the point of view of the cut Hankel transformation. In the last two sections which form a kind of appendix and have but little connection with the rest of the paper, I give a biorthogonal set consisting of Laguerre polynomials (for $\mathscr{R}(a)<-1)$ and of what I would call cut Laguerre polynomials, both multiplied by suitable biorthogonalising factors. The properties of this set can be derived, also by means of partial integration, from the corresponding properties of Laguerre's orthogonal set, or else from the generating functions. Here I choose the second way.

Throughout the paper I restrict myself to functions of the class $L^{2}(0, \infty)$.

\section{Hankel transforms and their reciprocal functions.}

2. Hankel's transformation of order $a I$ write in the form

$$
g(x)=\mathscr{H}_{a}\{f(x)\} \equiv \underset{Y \rightarrow \infty}{\operatorname{li} \text {. m. }} \int_{0}^{Y} J_{a}\{2 \sqrt{ }(x y)\} f(y) d y,[\mathcal{R}(a)>-1]
$$

The usual notation is slightly different, namely

$$
g_{1}(x)=\int_{0}^{\infty} J_{a}(x y)(x y)^{1} f_{1}(y) d y,
$$

probably because this reduces readily to the Fourier sine or cosine transform when $a= \pm \frac{1}{2}$ respectively. It seems, however, that in the general case, $a \neq \pm \frac{1}{2},(1)$ is simpler from the formal point of view, perhaps because $z^{-a} J_{a}(z)$ is a power-series in $z^{2}$.

The best known set of reciprocal functions of $\mathscr{H}_{a}$ is Laguerre's orthogonal set consisting of the functions

$$
\psi_{n}^{(\alpha)}(x)=e^{-x} x^{\frac{1}{a}} L_{n}^{(\alpha)}(2 x) \quad(n=0,1,2, \ldots) .
$$


(i) The functions $\psi_{n}^{(a)}$ belonging to even or odd values of $n$ are self or skew reciprocal respectively. Thus

$$
\psi_{n}^{(a)}=(-)^{n} \theta t_{a}\left\{\psi_{n}^{(a)}\right\}, \quad[\mathscr{R}(a)>-1] .
$$

(ii) If $\delta_{m n}$ is Kronecker's delta, being zero when $m \neq n$ and unity when $m=n$,

$$
\int_{0}^{\infty} \psi_{m}^{(a)}(x) \psi_{n}^{(a)}(x) d x=\frac{\Gamma(a+n+1)}{2^{a+1} n !} \delta_{m n}, \quad[\mathscr{R}(a)>-1] .
$$

Thus $\left\{\psi_{n}^{(a)}, \psi_{n}^{(a)}\right\}$ is a biorthogonal sequence and, in particular, for real values of $\alpha, a>-1,\left\{\psi_{n}^{(a)}\right\}$ is an orthogonal sequence.

(iii) The system $\left\{\psi_{n}^{(a)}\right\}$ is complete and closed in $L^{2}(0, \infty)$ if $\mathscr{R}(a)>-1$.

All these properties are very well known. Perhaps not equally well known is a generalisation of (3), namely ${ }^{1}$

(5) $k^{n} e^{-x} x^{1 \alpha} L_{n}^{(a)}\left(\frac{x}{k}\right)=(-)^{n} g f_{a}\left\{(1-k)^{n} e^{-x} x^{\frac{1}{a} a} L_{n}^{(a)}\left(\frac{x}{1-k}\right)\right\}, \quad[\mathscr{R}(a)>-1]$.

(3) corresponds to $k=\frac{1}{2}$ in (5). Replacing $x$ and $y$ in (5) by $2 k x$ and $2(1-k) y$ respectively we get

$$
e^{-x} x^{\frac{1}{a} a} L_{n}^{(a)}(2 x)=c_{n, k} \int_{0}^{\infty} K_{a, k}(x, y) e^{-y} y^{\frac{1}{a} a} L_{n}^{(a)}(2 y) d y,
$$

where and

$$
K_{a, k}(x, y)=2 k^{-\frac{i}{d} a}(1-k)^{\frac{1}{2} a+1} e^{-(1-2 k)(x+y)} J_{a}\{4 \sqrt{x y k(1-k)}\}
$$

$$
c_{n, k}=\left(1-\frac{1}{k}\right)^{n}
$$

Kober ${ }^{2}$ interpreted this equation by saying that $\psi_{n}^{(a)}$ is also an eigenfunction with respect to the kernel $K_{a, k}$, the corresponding eigen-value being $c_{n, k}$.

In what follows we shall make use of, and generalise, the generating function of Laguerre polynomials

$$
\sum_{n=0}^{\infty} u^{n} L_{n}^{(a)}(z)=(1-u)^{-i-1} e^{-u z /(1-u)},(|u|<1)
$$

1 A. Erdélyi, Quart. J. of Math. (Oxford Series) 9 (1938), 196-8 (8).

${ }^{2}$ H. Kober, Quart. J. of Math. (Oxford Series) 10 (1939), 45.59, especially section 8. Kober takes $k=\frac{1}{2}(1+i \cot \pi r), r$ real and not integral. 
and the following representations :

$$
L_{n}^{(a)}(z)=\frac{\Gamma(a+n+1)}{n ! \Gamma(a+1)}{ }_{1} F_{1}(-n ; a+1 ; z)=\sum_{r=0}^{n}\left(\begin{array}{l}
n+a \\
n-r
\end{array}\right) \frac{(-z)^{r}}{r !}
$$

and

$$
L_{n}^{(a)}(x)=\frac{e^{x} x^{-a}}{n !} \frac{d^{n}\left(e^{-x} x^{a+n}\right)}{d x^{n}}
$$

3. When $\mathscr{R}(a)<-1$, then the Bessel function has to be replaced by the cut Bessel function of the first kind

$$
J_{a, m}(z)=\sum_{r=m}^{\infty}(-)^{r}\left(\frac{1}{2} z\right)^{a+2 r} \Gamma(a+r+1), \quad(m=0,1,2, \ldots),
$$

and the ordinary Hankel transform by

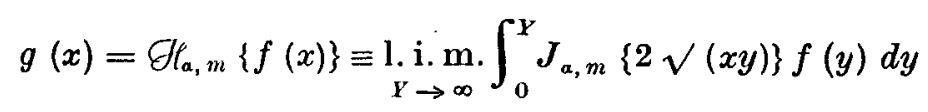

where $m=0$ if $\mathscr{R}(\alpha)>-1$; otherwise $m$ is chosen so that $-1<\mathscr{R}(a)+2 m<1$. Negative odd integral values of $a$ are excluded by this convention 1 .

The two-fold reduction of $\theta t_{a, m}$ to $\partial l_{a+2 m}$ by means of repeated integration by parts yields the following theorem:

Let $-1<\mathscr{R}(\alpha)+2 m<1, m=1,2,3, \ldots ;$ let $F(x)$ and $G(x)$ belong to $L^{2}(0, \infty)$ and

$$
G(x)=\theta t_{a+2 m}\{F(x)\}
$$

Then

when either

$$
g(x)=\mathscr{f} f_{a, m}\{f(x)\}
$$

or

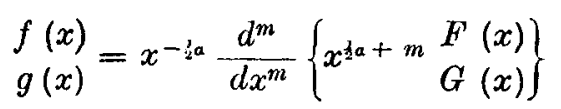

With (11) the additional assumption is necessary that $f$ (and consequently g) exists and belongs to $L^{2}(0, \infty)$. With (12) no additional assumption is necessary.

1 The cut Hankel transform is mentioned in E. C. Titchmarsh's Introduction to the theory of Fourier integrals (Oxford, 1937), § 8.4 Example (1); a full discussion of $\mathscr{f l}_{a, m}$ was given by H. Kober, Quart. J. of Math. (Oxford Series) 8(1937), 186-99. 
The proof of this theorem has been published in a joint note by Dr Kober and myself ${ }^{1}$. Here I only remark that analogous statements hold true for the ordinary Hankel transform, $\mathcal{R}(a)>-1$, and that in this latter case fractional integration by parts may be used yielding fractional derivatives and integrals instead of (11) and (12). The reader will easily see the connection with the general rules in Titchmarsh's Fourier Transforms, §§ 9.14-9.16.

Now, from (3)

$$
\psi_{n}^{(a+2 m)}(x)=(-)^{n} \mathscr{H}_{a+2 m}\left\{\psi_{n}^{(a+2 m)}(x)\right\} .
$$

Hence if we define

$$
\chi_{n}^{(a, m)}(x)=\frac{x^{-\frac{1}{2} a}}{m !} \frac{d^{m}}{d x^{m}}\left\{x^{\jmath a+m} \psi_{n}^{(a+2 m)}(x)\right\},
$$

and

$$
\phi_{n}^{(a, m)}(x)=\frac{x^{\underline{j} a}}{(m-1) !} \int_{0}^{x}(x-y)^{m-1} y^{-\underline{\underline{k} a-m}} \psi_{n}^{(a+2 m)}(y) d y,
$$

then

$$
\chi_{n}^{(a, m)}(x)=(-)^{n} \mathscr{H}_{a, m}\left\{\chi_{n}^{(a, m)}(x)\right\}, \quad(n=0,1,2, \ldots)
$$

and

$$
\phi_{n}^{(a, m)}(x)=(-)^{n} \mathscr{H}_{a, m}\left\{\phi_{n}^{(a, m)}(x)\right\}, \quad(n=0,1,2, \ldots)
$$

if only

$$
-1<\mathscr{Q}(\alpha)+2 m<1, \quad m=1,2,3, \ldots \ldots
$$

Plainly the conditions of validity of the rule stated above are fulfilled, for $\psi_{n}^{(a+2 m)}(x)$ belongs to $L^{2}(0, \infty), \chi_{n}^{(a, m)}(x)$ exists and belongs to $L^{2}(0, \infty)$.

Neither of the sequences $\left\{\chi_{n}^{(a, m)}\right\},\left\{\phi_{n}^{(a, m)}\right\}$ is an orthogonal one; that is, in general, neither of the integrals

$$
\int_{0}^{\infty} \chi_{n}^{(a, m)} \chi_{n}^{(a, m)} d x, \quad \int_{0}^{\infty} \phi_{n}^{(a, m)} \phi_{n^{\prime}}^{(a, m)} d x
$$

vanishes whenever $n \neq n^{\prime}$. Of course both of them vanish when $n$ is even and $n^{\prime}$ is odd (or conversely), for then the two functions involved belong to different eigen-values. Also the first integral can be shown to vanish whenever $\left|n-n^{\prime}\right|>m$. For $m=0$ this gives the orthogonality property of $\left\{\psi_{n}^{(\alpha)}\right\}$.

1 Qhaart. J. of Math. (Oxford Series) 11 (1940), 212.21, Theorems 3 and 4. 
All these facts will not be dealt with in detail because they are of minor importance. More important is it that $\left\{\chi_{n}^{(a, m)}, \phi_{n}^{(a, m)}\right\}$ is a biorthogonal set, i.e. that the integral

$$
\int_{0}^{\infty} \chi_{n}^{(a, m)} \phi_{n^{\prime}}^{(a, m)} d x
$$

vanishes whenever $n \neq n^{\prime}$. This will be shown in section 10 .

\section{The polynomials $L$.}

4. First I shall deal with the system $\left\{\chi_{n}^{(a, m)}\right\}$. Corresponding to (2) I put

Then, from (13) and (2),

$$
\chi_{n}^{(a, m)}(x)=e^{-x} x^{1-\alpha+m} L_{n+m}^{(a, m)}(2 x) .
$$

$$
L_{n+m}^{(a, m)}(2 x)=\frac{e^{x} x^{-a-m}}{m !} \frac{d^{m}}{d x^{m}}\left\{e^{-x} x^{a+2 m} L_{n}^{(a+2 m)}(2 x)\right\} .
$$

Plainly $L_{n+m}^{(a, m)}(2 x)$ is a polynomial of degree $n+m$ in $x$.

Regarding the formal properties, it is equally easy to deal with the slightly generalised polynomial

$$
L_{n, m}^{(a+m)}(a x, b x)=\frac{e^{b x} x^{-a-m}}{m !} \frac{d^{m}}{d x^{m}}\left\{e^{-b x} x^{a+2 m} L_{n}^{(a+2 m)}(a x)\right\} .
$$

It is easily seen that this is a polynomial in the variables $a x$ and $b x$ of respective degrees $n$ and $m$, the coefficients of which are independent of $a, b$ and $x$.

Now put

$$
\chi_{n}^{(a, m)}(x ; k)=k^{n} e^{-x} x^{\frac{j}{a} a+m} L_{n, m}^{(a+m)}\left(\frac{x}{k}, x\right) .
$$

Then it will be shown that

$$
\chi_{n}^{(a, m)}(x ; k)=(-)^{n} \oiiint_{a, m}\left\{\chi_{n}^{(a, m)}(x ; 1-k)\right\},[-1<\mathcal{R}(a)+2 m<1] .
$$

This is the generalisation of $(5)$.

Proof: $-\mathcal{R}(a)+2 m$ being larger than -1 , from $(5)$.

$$
k^{n} e^{-x} x^{\frac{1}{a}+m} L_{n}^{(a+2 m)}\left(\frac{x}{k}\right)=(-)^{n} \mathscr{H}_{a+2 m}\left\{(1-k)^{n} e^{-x} x^{\jmath a+m} L_{n}^{(a+2 m)}\left(\frac{x}{1-k}\right)\right\} \text {, }
$$

and the functions on both sides of this belong to $L^{2}(0, \infty)$. Hence from (11)

$$
\begin{aligned}
& k^{n} x^{-\frac{1}{2} a} \frac{d^{m}}{d x^{m}}\left[e^{-x} x^{a+2 m} L_{n}^{(a+2 m)}\left(\frac{x}{k}\right)\right] \\
& \quad=(-)^{n} \mathscr{H}_{a, m}\left\{(1-k)^{n} x^{-\frac{1}{2} a} \frac{d^{m}}{d x^{m}}\left[e^{-x} x^{a+2 m} L_{n}^{(a+2 m)}\left(\frac{x}{1-k}\right)\right]\right\},
\end{aligned}
$$


and this is, by definitions (19) and (20), identical with (21). For $k=\frac{1}{2},(21)$, except for a constant factor, reduces to (15).

5. In this section it will be shown that the polynomial defined by (19) is identical with the polynomial I denoted by the same symbol and studied a few years ago.

In what follows I constantly use the notation

Since

$$
(a)_{n}=\frac{\Gamma(\alpha+n)}{\Gamma(\alpha)}
$$

from (19) and (7),

$$
{ }_{1} F_{1}(-n ; a+1 ; z)=\sum_{r=0}^{n} \frac{(-n)_{r} z^{r}}{r !(a+1)_{r}},
$$

$$
\begin{aligned}
L_{n, m}^{(a+m)}(a x, b x) & =\frac{(a+2 m+1)_{n}}{m ! n !} e^{b x} x^{-a-m} \times \\
& \sum_{r=0}^{n} \frac{(-n)_{r} a^{r}}{r !(a+2 m+1)_{r}} \frac{d^{m}}{d x^{m}}\left(e^{-b x} x^{a+2 m+r}\right), \\
& =\frac{(a+2 m+1)_{n}}{n !} \sum_{r=0}^{n} \frac{(-n)_{r}(a x)^{r}}{r !(a+2 m+1)_{r}} L_{m}^{(a+m+r)}(b x),
\end{aligned}
$$

by (8). Using again (7), now for $L_{m !}^{(a+m+r)}(b x)$, we see that

Hence

$$
\frac{(a+2 m+1)_{n}}{(\alpha+2 m+1)_{r}} \frac{(\alpha+m+r+1)_{m}}{(a+m+r+1)_{s}}=\frac{(a+m+1)_{m+n}}{(\alpha+m+1)_{r+s}} .
$$

$$
\begin{aligned}
L_{n, m}^{(a+m)}(a x, b x) & =\frac{(a+m+1)_{m+n}}{m ! n !} \sum_{r=0}^{n} \sum_{s=0}^{m} \frac{(-n)_{r}(-m)_{s}(a x)^{r}(b x)^{s}}{r ! s !(a+m+1)_{r+s}} \\
& =\frac{(a+m+1)_{m+n}}{m ! n !} \Phi_{2}(-n,-m ; a+m+1 ; a x, b x)
\end{aligned}
$$

in the notation of Professor Humbert'. This is exactly the definition: of what I called at that time Laguerre polynomials of two variables ${ }^{2}$.

6. It is now only too easy to derive a vast number of formal relations, such as recurrence formulae, series, etc., for the polynomials $L_{n, m}^{(a+m)}$ either from the corresponding properties of Laguerre polynomials by means of (19), or else from my earlier work ${ }^{3}$. In this

1 P. Humbert, Proc. Royal Soc., Edinburgh, 41 (1920/21), 73-96. See also P. Appell et J. Kampé de Fériet, Fonctions hypergéométriques. Polynomes d'Hermite. (Paris, 1926), p. 126.

2 A. Erdélyi, Wiener Sitzungsberichte, 146 (1937), 431-67 (11, 3).

3 Abschnitt III of the paper quoted in the preceding footnote. 
section I will write down only those few formal relations which exhibit the connection between $L_{n, m}^{(a+m)}$ and ordinary Laguerre polynomials and / or are needed in subsequent work.

Let us begin with the generating function of these polynomials. From (19)

$$
\begin{gathered}
\sum_{n=0}^{\infty} u^{n} L_{n, m}^{(a+m)}(a x, b x)=\frac{1}{m !} e^{l x} x^{-a-m} \frac{d^{m}}{d x^{m}}\left\{e^{-b x} x^{a+2 m} \sum_{n=0}^{\infty} u^{n} L_{n}^{(a+2 m)}(a x)\right\} \\
=\frac{e^{b x} x^{-a-m}}{m !(1-u)^{a+2 m+1}} \frac{d^{m}}{d x^{m}}\left\{e^{-\{b+a u /(1-u)\} x} x^{a+2 m}\right\},
\end{gathered}
$$

from (6). For $|u|<1$ the series is absolutely and uniformly convergent in any finite interval $0<x_{1} \leqq x \leqq x_{2}$ and so term-by-term differentiation is permissible. Finally, by (8), this last result can be expressed by the Laguerre polynomial of degree $m$, giving

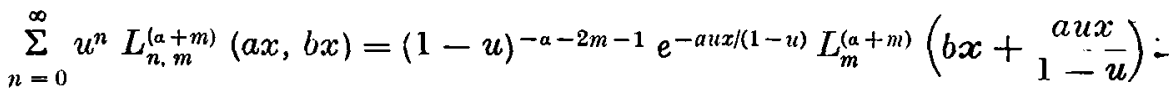

This is the generating function of our polynomials.

From (19) and (8) we have

$$
L_{n, m}^{(a+m)}(a x, b x)=\frac{e^{b x} x^{-a-m}}{m ! n !} \frac{d^{m}}{d x^{m}}\left\{e^{(a-b) x} \frac{d^{n}}{d x^{n}}\left(e^{-a x} x^{a+2 m+n}\right)\right\} .
$$

From this representation the following reduction formulae presently follow

$$
\begin{aligned}
L_{n, m}^{(a+m)}(a x, 0) & =\frac{(a+m+n+1)_{m}}{m !} L_{n}^{(a+m)}(a x), \\
L_{n, m}^{(a+m)}(0, b x) & =\frac{(a+2 m+1)_{n}}{n !} L_{m}^{(a+m)}(b x), \\
L_{n, m}^{(a+m)}(a x, a x) & =\frac{(m+n) !}{m ! n !} L_{n+m}^{(a+m)}(a x), \\
L_{n, 0}^{(a)}(a x, b x) & =L_{n}^{(a)}(a x), \\
L_{0, m}^{(a+m)}(a x, b x) & =L_{m}^{(a+m)}(b x) .
\end{aligned}
$$

Comparing (18) and (19), .

$$
L_{n+m}^{(a, m)}(2 x)=L_{n, m}^{(a+m)}(2 x, x) .
$$

Finally, from the expansion of section 5 , it is readily seen that

$$
\lim _{a \rightarrow \infty}\left[a^{-n} L_{n, m}^{(a+m)}(a x, b x)\right]=\frac{(-x)^{n}}{n !} L_{m}^{(a+m+n)}(b x) .
$$


This last relation will immediately be required in proving the formula

$$
\begin{gathered}
\frac{m !}{(n+m) !} e^{-x} x^{\frac{1}{2} a+m+n} L_{m}^{(a+m+n)}(x)=g t_{a, m}\left\{e^{-x} x^{\frac{1}{2} a+m} L_{n+m}^{(a+m)}(x)\right\}, \\
{[-1<\mathscr{R}(a)+2 m<1] .}
\end{gathered}
$$

which is really only the limiting case $k \rightarrow 0$ of (21). For, from (20) and (26),

$$
\begin{aligned}
\chi_{n}^{(a, m)}(x ; 0) & =e^{-x} x^{j a+m} \lim _{k \rightarrow 0}\left[k^{n} L_{n, m}^{(a+m)}\left(\frac{x}{k}, x\right)\right] \\
& =\frac{(-)^{n}}{n !} e^{-x} x^{j a+m+n} L_{m}^{(a+m+n)}(x),
\end{aligned}
$$

and according to (20) and the third equation (24),

$$
\chi_{n}^{(\alpha, m)}(x ; 1)=\frac{(m+n) !}{m ! n !} e^{-x} x^{\frac{1}{2} a+m} L_{n+m}^{(a+m)}(x) .
$$

Hence (21) with $k=0$ yields (27).

7. In this section the Laplace and Mellin transforms of our reciprocal functions will be evaluated. In order to do so, I begin with the slightly more general integral

$$
\int_{0}^{\infty} e^{-c x} x^{\sigma-1} L_{n, m}^{(a+m)}(a x, b x) d x, \quad \mathcal{R}(\sigma)>0, \mathscr{R}(c)>0 .
$$

This is, using the expansion of section 5, equal to

$$
\begin{aligned}
& \frac{(a+m+1)_{m+n}}{m ! n !} \sum_{r=0}^{n} \sum_{s=0}^{m} \frac{(-n)_{r}(-m)_{s} a^{r} b^{s}}{r ! s !(a+m+1)_{r+8}} \int_{0}^{\infty} e^{-c x} x^{\sigma+r+s-1} d x \\
& =\frac{\Gamma(\sigma)(a+m+1)_{m+n}}{m ! n ! c^{\sigma}} \sum_{r=0}^{n} \sum_{s=0}^{m} \frac{(-r)_{r}(-m)_{s}(\sigma)_{r+s}}{r ! s !(a+m+1)_{r+s}}\left(\frac{a}{c}\right)^{r}\left(\frac{b}{c}\right)^{s} .
\end{aligned}
$$

Hence by Appell's definition of the hypergeometric series $F_{1}$ of two variables ${ }^{1}$

$$
\begin{aligned}
& \int_{0}^{\infty} e^{-c x} x^{\sigma-1} L_{n, m}^{(a+m)}(a x, b x) d x=\Gamma(\sigma) \frac{(a+m+1)_{m+n}}{m ! n ! c^{\sigma}} \times \\
& \quad F_{1}\left(\sigma ;-n,-m ; a+m+1 ; \frac{a}{c}, \frac{b}{c}\right), \quad[-\mathcal{R}(\sigma)>0,-\mathcal{R}(c)>0] .
\end{aligned}
$$

'Appell et Kampé de Fériet, l.c., p. 14 (11). See also E. 'T. Whittaker and G. N. Watson, Modern Analysis (Cambridge, 1927), p. 300, Example 22. 
From the derivation of (28) it is easily seen that

$$
\begin{gathered}
\int_{0}^{\infty} e^{-c x} x^{a+m} L_{n, m}^{(a+m)}(a x, b x) d x=\frac{\Gamma(a+2 m+n+1)}{m ! n ! c^{a+m+1}}\left(1-\frac{a}{c}\right)^{n}\left(1-\frac{b}{c}\right)^{m} . \\
{[\mathcal{R}(a+m)>-1, \quad \mathcal{R}(c)>0] .}
\end{gathered}
$$

Another particular case of (28) will be important when obtaining the Mellin transform of the reciprocal functions of the cut Hankel transform. This particular case arises when $b=c$. According to Appell et Kampé·de Fériet, l.c., p. 22 (23),

$$
F_{1}\left(\sigma ;-n,-m ; a+m+1 ; \frac{a}{b}, 1\right)=\frac{(a+m-\sigma+1)_{m}}{(a+m+1)_{m}} F\left(\sigma,-n ; a+2 m+1 ; \frac{a}{b}\right)
$$

hence

$$
\begin{gathered}
\int_{0}^{\infty} e^{-b x} x^{\sigma-1} L_{n . m}^{(a+m)}(a x, b x) d x \\
(30)=\frac{\Gamma(\sigma)(a+2 m+1)_{n}(a+m-\sigma+1)_{m}}{m ! n ! b^{\sigma}} F\left(\sigma,-n ; a+2 m+1 ; \frac{a}{b}\right), \\
{[\Omega(\sigma)>0, \Omega(b)>0] .}
\end{gathered}
$$

A similar formula exists for $a=c$.

Now we shall obtain the Mellin transform of $\chi_{n}^{(a, m)}(x)$ and by this we shall prove once more the self-reciprocal property of $\chi_{n}^{(a, m)}(x)$. Put

Then, from (17) and (25),

$$
\mathrm{X}_{n}^{(\alpha, m)}(t)=\int_{0}^{\infty} \chi_{n}^{(a, m)}(x) x^{-i+\frac{1}{2} i t} d x .
$$

$$
\mathrm{X}_{n}^{(\alpha, m)}(t)=\int_{0}^{\infty} e^{-x} x^{\underline{1} a+m-\frac{1}{2}+\underline{1} i t} L_{n, m}^{(\alpha+m)}(2 x, x) d x,
$$

and, from (30), this is equal to

$$
\begin{aligned}
& \mathrm{X}_{n}^{(a, m)}(t)=\frac{(a+2 m+1)_{n}}{m ! n !} \frac{\Gamma\left(\frac{1}{2} a+m+\frac{1}{2}+\frac{1}{2} i t\right) \Gamma\left(\frac{1}{2} a+m+\frac{1}{2}-\frac{1}{2} i t\right)}{\Gamma\left(\frac{1}{2} a+\frac{1}{2}-\frac{1}{2} i t\right)} \times \\
& F\left(-n, \frac{1}{2} \alpha+m+\frac{1}{2}+\frac{1}{2} i t ; a+2 m+1 ; 2\right) \text {. }
\end{aligned}
$$

The integral is certainly convergent for purely real values of $t$. It is even convergent in the half-plane $\mathscr{R}\left(\frac{1}{2} a+m+\frac{1}{2}+\frac{1}{2} i t\right)>0$.

By Euler's transformation,

we have also

$$
F(\alpha, \beta ; \gamma ; z)=(1-z)^{-a} F(a, \gamma-\beta ; \gamma ; z)
$$

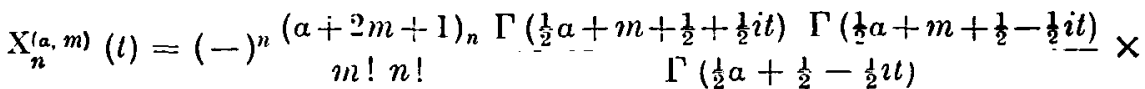

$$
\begin{aligned}
& F\left(-n, \frac{1}{2} a+m+\frac{1}{2}-\frac{1}{2} i l ; a+2 m+1 ; 2\right) \text {, }
\end{aligned}
$$


and hence

$$
\Gamma\left(\frac{a+1-i t}{2}\right) X_{n}^{(a, m)}(t)=(-)^{n} \Gamma\left(\frac{a+1+i t}{2}\right) X_{n}^{(a, m)}(-t),
$$

and this relation is exactly equivalent to (15). Thus this is a second proof of (15), quite independent of the general theorem of section 3.

When trying to obtain the Laplace transform of the reciprocal functions, the difficulty arises that (29) is only convergent for $\mathcal{R}(a+m)>-1$. But with cut Hankel transforms, we have $\mathcal{R}(a+m)<-1$, unless $-2<\mathcal{R}(a)<-1$; so in general the ordinary Laplace transform will not work. In order to get convergent integrals one has to "cut" the kernel of the Laplace transform too, that is use instead of the exponential function the "cut" exponential function

$$
E_{m}(x)=\sum_{r=m}^{\infty} \frac{(-x)^{r}}{r !}=\frac{(-x)^{m}}{m !}{ }_{1} F_{1}(1 ; m+1 ;-x),
$$

which behaves for small values of $x$ like $(-x)^{m} / m$ !, thus bringing the index of the power of $x$ to $a+2 m$, the real part of which certainly exceeds -1 .

Consequently it is natural to investigate

$$
\int_{0}^{\infty} E_{m}(s x) x^{1 / \alpha} \chi_{n}^{(\alpha, m)}(x) d x
$$

which is, according to (13), equal to

$$
\frac{1}{m !} \int_{0}^{\infty} E_{m}(s x) \frac{d^{m}}{d x^{m}}\left\{x^{j a+m} \psi_{n}^{(a+2 m)}(x)\right\} d x .
$$

Integrating $\dot{m}$ times by parts, this becomes, on account of

and using (2),

$$
\frac{d^{m} E_{m}(s x)}{d x^{m}}=(-s)^{m} e^{-8 x}
$$

$$
\frac{s^{m}}{m !} \int_{0}^{\infty} e^{-s x} x^{j a+m} \psi_{n}^{(a+2 m)}(x) d x=\frac{s^{m}}{m !} \int_{0}^{\infty} e^{-(s+1) x} x^{a+2 m} L_{n}^{(a+2 m)}(2 x) d x .
$$

Now it is possible to use (29) with $a=2, b=0, c=s+1$, and thus to obtain

$$
\begin{gathered}
\int_{0}^{\infty} E_{m}(s x) x^{\frac{1}{a} a} x_{n}^{(a, m)}(x) d x=\frac{\Gamma(a+2 m+n+1) s^{m}(s-1)^{n}}{m ! n !(s+1)^{a+2 m+n+1}-} \\
{[-1<\mathcal{R}(a)+2 m<1 ; \mathscr{R}(s)>-1] .}
\end{gathered}
$$

This is the proper generalisation of the familiar formula for the Laplace transform of $\psi_{n}^{(a)}(x)$. Also (32) can be used to prove (15). 


\section{The functions $\Lambda$.}

8. After having discussed the functions $\chi_{n}^{(a, m)}$ and the polynomials $L_{n, m}^{(a+m)}$ in some detail, I may confine myself to a short review of the corresponding results for the functions $\phi_{n}^{(a, m)}$ without giving proofs and without going into details.

Corresponding to (17), I put

$$
\phi_{n}^{(a . m)}(x)=e^{-x} x^{\frac{1 a+m}{a}} \Lambda_{n+m}^{(a, m)}(2 x)
$$

where, from (14) and (2),

$$
\Lambda_{n+m}^{(a, m)}(2 x)=\frac{x^{-m}}{(m-1) !} \int_{0}^{x}(x-y)^{m-1} e^{x-y} L_{n}^{(a+2 n)}(2 y) d y .
$$

Instead of this function I introduce

$$
\Lambda_{n, m}^{(a+m)}(a x, b x)=\frac{x^{-m}}{(m-1) !} \int_{0}^{x}(x-y)^{m-1} e^{b(x-y)} L_{n}^{(a+2 m)}(a y) d y
$$

and form

$$
\phi_{n}^{(a, m)}(x ; k)=k^{n} e^{-x} x^{j a+m} \Lambda_{n, m}^{(a+m)}\left(\frac{x}{k}, x\right)
$$

which satisfies the important relation

$$
\begin{gathered}
\phi_{n}^{(a, m)}(x ; k)=(-)^{n} \mathscr{l}_{a, m}\left\{\phi_{n}^{(a, m)}(x ; 1-k)\right\}, \\
{[-1<\mathscr{R}(a)+2 m<1] .}
\end{gathered}
$$

The proof of (37) is similar to that of (21). Start with (5) replacing $a$ by $a+2 m$, and apply the general theorem of section 3 with (12). The particular case $k=\frac{1}{2}$ of $(37)$ is (16); the limiting cases $k \rightarrow 0$ and $k \rightarrow 1$, inversions of each other, are easily obtained.

Also $\Lambda_{n, m}^{(a+m)}(a x, b x)$ can be expressed by a confluent hypergeometric function of the two variables $a x$ and $b x$, the only difference compared to the results of section 5 being that the series obtained is terminating only in $a x$, but an infinite series in $b x$. Thus $\Lambda_{n, m}^{(a+m)}$ is a polynomial of degree $n$ in $a x$, but has, in $b x$, rather the character of the exponential function $e^{b x}$. Using the notation of Appell et Kampé de Fériet, l. c., p. 150,

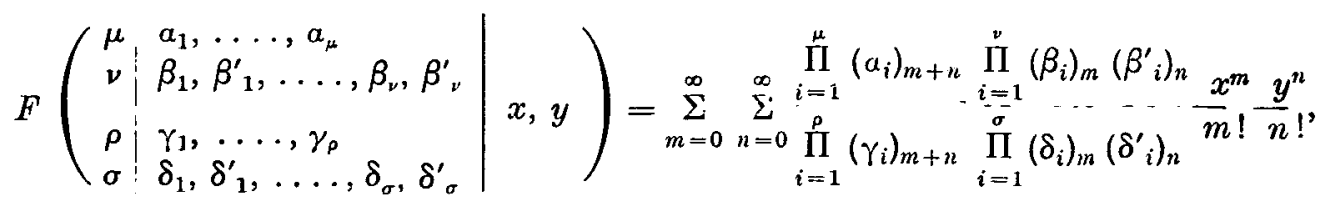


we find

$$
\Lambda_{n, m}^{(\alpha+m)}(x, y)=\frac{(\alpha+2 m+1)_{n}}{m ! n !} F\left(\begin{array}{l|l}
0 & . \\
2 & -n, m ; 1, \beta \\
1 & m+1 \\
1 & \alpha+2 m+1, \beta
\end{array} \mid a x, b x\right) .
$$

Here $\beta$ is a dummy parameter which cancels in the series.

The generating function of the $\Lambda_{n, m}^{(a+m)}$ reads

$$
\begin{aligned}
& \sum_{n=0}^{\infty} u^{n} \Lambda_{n, m}^{(a+m)}(a x, b x)=(1-u)^{-a-2 m-1} \times \\
& \quad e^{b x}\left(-b x-\frac{a u x}{1-u}\right)^{-m} E_{m}\left(b x+\frac{a u x}{1-u}\right), \quad|u|<1 .
\end{aligned}
$$

Here $E_{m}$ is again the "cut" exponential function defined in the preceding section.

Since

$$
\Lambda_{n+m}^{(a, m)}(2 x)=\Lambda_{n, m}^{(a+m)}(2 x, x),
$$

all the relations simplify considerably for the reciprocal functions.

9. In order to find the Mellin transform of $\phi_{n}^{(a, m)}$ and what corresponds to the Laplace transform in this case, one may again start from the integral

$$
\begin{aligned}
& \int_{0}^{\infty} e^{-c x} x^{\sigma-1} \Lambda_{n, m}^{(\alpha+m)}(a x, b x) d x \\
& =\frac{\Gamma(\sigma)(a+2 m+1)_{n}}{m ! n ! c^{\sigma}} F\left(\begin{array}{r|l|l}
1 & \alpha \\
2 & -n, m ; 1, \beta \\
1 & \begin{array}{l}
\alpha+1 \\
1
\end{array} & \frac{a}{c}, \frac{b}{c}
\end{array}\right), \\
& {[\mathcal{R}(a)>0, \mathscr{R}(c)>|\mathscr{R}(b)|] . .}
\end{aligned}
$$

Two particular cases are worth mentioning:

$$
\begin{aligned}
& \int_{0}^{\infty} e^{-c x} x^{m} \Lambda_{n, m}^{(a+m)}(a x, b x) d x \\
&= \frac{(a+2 m+1)_{n}}{n ! c^{m+1}}\left(1-\frac{b}{c}\right)^{-m} F\left(-n, 1 ; a+2 m+1 ; \frac{a}{c}\right), \\
& {[\mathcal{R}(c)>|\mathcal{R}(b)|], }
\end{aligned}
$$

and the limiting case

$$
\begin{gathered}
\int_{0}^{\infty} e^{-b x} x^{\sigma-1} \Lambda_{n, m}^{(a+m)}(a x, b x) d x \\
=\frac{(-)^{m}}{n !}(a+2 m+1)_{n} \Gamma(\sigma-m) b^{-\sigma} F\left(-n, \sigma ; a+2 m+1 ; \frac{a}{b}\right), \\
{[0<\mathcal{R}(\sigma)<1, \mathcal{R}(b)>0] .}
\end{gathered}
$$


This limiting case is used when computing the Mellin transform of $\phi_{n}^{(a, m)}(x)$, for real values of $t$,

$$
\begin{gathered}
\Phi_{n}^{(a, m)}(t) \equiv \int_{0}^{\infty} \phi_{n}^{(a, m)}(x) x^{-1+i t} d x \\
=\frac{(-)^{m}}{n !}(\alpha+2 m+1)_{n} \Gamma\left(\frac{a+1+i t}{2}\right) F\left(-n, \frac{a+2 m+1+i t}{2} ; a+2 m+1 ; 2\right) \\
{[-1<\mathcal{R}(\alpha)+2 m<1] .}
\end{gathered}
$$

Again, using Euler's transformation of the hypergeometric polynomial on the right of (44), it is readily shown that

$$
\Gamma\left(\frac{a+1-i t}{2}\right) \Phi_{n}^{(a, m)}(t)=(-)^{n} \Gamma\left(\frac{a+1+i t}{2}\right) \Phi_{n}^{(a, m)}(-t),
$$

and from this the reciprocal property of the $\phi_{n}^{(a, m)}(x)$ follows.

In the case of the system $\left\{\phi_{n}^{(a, m)}\right\}$ the Laplace transform has not to be "cut," but, on the contrary, extended; the right kernel is $e^{-8 x} L_{m}^{(a+m)}(s x)$ instead of the $e^{-8 x}$ of the ordinary Laplace transform and the $E_{m}(s x)$ of the "cut" one which has been used in section 7 .

The formula corresponding to (32) now reads

$$
\begin{gathered}
\int_{0}^{\infty} e^{-s x} L_{m}^{(a+m)}(s x) x^{1 a+m} \phi_{n}^{(\alpha, m)}(x) d x=\frac{(-)^{m} \Gamma(\alpha+2 m+n+1)(s-1)^{n}}{m ! n !(s+1)^{\alpha+2 m+n+1}}, \\
{[-1<\mathcal{R}(a)+2 m<1 ; \mathcal{R}(s)>1] .}
\end{gathered}
$$

This can be proved in a similar way to the proof of $(32)$ by reducing the integral by $m$ repeated partial integrations to the familiar one representing the Laplace transform of $x^{\frac{1}{a} a+m} \psi_{n}^{(a+2 m)}(x)$.

\section{The biorthogonal system $\{\chi, \phi\}$.}

10. In this section it will be proved that $\left\{\chi_{n}^{(a, m)}, \phi_{n}^{(a, m)}\right\}$ is a biorthogonal system, that is, that the integral

$$
\int_{0}^{\infty} \chi_{n}^{(\alpha, m)} \phi_{n^{\prime}}^{(a, m)} d x
$$

vanishes unless $n=n^{\prime}$.

The proof is very simple, for from (13) it follows, by $m$ subsequent integrations by parts, that

$$
\begin{gathered}
\int_{0}^{\infty} \chi_{n}^{(a, m)}(x) \phi_{n^{\prime}}^{(a, m)}(x) d x=\frac{1}{m !} \int_{0}^{\infty} \frac{d^{m}}{d x^{m}}\left\{x^{\frac{1}{3} a+m} \psi_{n}^{(a+2 m)}\right\} x^{-\frac{1}{2} a} \phi_{n}^{(a, m)} d x \\
=\frac{(-)^{m}}{m !} \int_{0}^{\infty} x^{\frac{1}{2} a+m} \psi_{n}^{(a+2 m)} \frac{d^{m}}{d x^{m}}\left\{x^{-\frac{1}{2} a} \phi_{n^{\prime}}^{(a, m)}\right\} d x .
\end{gathered}
$$


The integrated terms cancel when $-1<\mathcal{R}(a)+2 m<1$. Now, from (14),

and hence

$$
\frac{d^{m}}{d x^{m}}\left\{x^{-\frac{1}{d a}} \phi_{n^{\prime}}^{(a, m)}\right\}=x^{-j a-m} \psi_{n^{\prime}}^{(a+2 m)}
$$

$$
\int_{0}^{\infty} \chi_{n}^{(a, m)} \phi_{n^{(a,}}^{(a)} d x=\frac{(-)^{m}}{m !} \int_{0}^{\infty} \psi_{n}^{(a+2 m)} \psi_{n}^{(a+2 m)} d x .
$$

Thus from the orthogonality of the system $\left\{\psi_{n}^{(a+2 m)}\right\}$ the biorthogonality of $\left\{\chi_{n}^{(a, m)}, \phi_{n}^{(a, m)}\right\}$ follows. Moreover, from (4),

$$
\int_{0}^{\infty} x_{n}^{(a, m)} \phi_{n^{\prime}}^{(a, m)} d x=\frac{(-)^{m} \Gamma(a+2 m+n+1)}{m ! n ! 2^{a+2 m+1}} \delta_{n n^{\prime}}, \quad[-1<\mathcal{R}(\alpha)+2 m<1] .
$$

In a paper "On some biorthogonal sets" I introduced" a generalisation of the present set which appears when in the definition of $\chi_{n}^{(a, m)}$ and $\phi_{n}^{(a, m)}$ fractional derivatives and integrals are used. The expansions of the functions, denoted there by $X_{n}$ and $\Phi_{n}$, into a terminating series of confluent hypergeometric functions of one variable only, apply to the present case too.

The biorthogonality of the system $\left\{\chi_{n}^{(a, m)}, \phi_{n}^{(a, m)}\right\}$ can be used in order to attach to every function of the class $L^{2}(0, \infty)$ a series in the $\chi_{n}^{(a, m)}$, namely

with coefficients

$$
f(x) \sim \sum_{n=0}^{\infty} c_{n} \chi_{n}^{(a, m)}(x)
$$

$$
c_{n}=\frac{(-)^{m} m ! n ! 2^{\alpha+2 m+1}}{\Gamma(\alpha+2 m+n+1)} \int_{0}^{\infty} f(x) \phi_{n}^{(a, m)}(x) d x .
$$

A function is self/skew reciprocal in the cut Hankel transform when all the $c_{n}$ with odd/even index $n$ respectively, corresponding to this function, vanish.

11. Now it will be proved that $\left\{\chi_{n}^{(\alpha, m)}, \phi_{n}^{(a, m)}\right\}$ is complete with respect to $L^{2}(0, \infty)$.

In order to do so one has to prove that, except for a nulfunction, there is no function in $L^{2}(0, \infty)$ with a nul-expansion; that is, if $f(x)$ belongs to $L^{2}(0, \infty)$ and all the integrals

$$
\int_{0}^{\infty} f(x) \phi_{n}^{(a, m)}(x) d x
$$

vanish, then $f(x)$ is a nul-function.

${ }^{1}$ Quart. J. of Math. (Oxford Series), 11 (1940), 111-123. 
Suppose that $f(x)$ is a function belonging to $L^{2}(0, \infty)$ such that

$$
\int_{0}^{\infty} f(x) \phi_{n}^{(e, m)}(x) d x=0, \quad[n=0,1,2, \ldots] .
$$

Then define

$$
F(x)=\frac{x^{-\frac{1}{2} a-m}}{(m-1) !} \int_{x}^{\infty}(y-x)^{m-1} y^{\frac{1}{2} a} f(y) d y .
$$

Obviously $F(x)$ exists for every value of $x>0$ and belongs to $L^{2}(0, \infty)$. Also, taking account of (14) and (50), we find by $m$ subsequent integrations by parts

$$
\int_{0}^{\infty} F \psi_{n}^{(a+2 m)} d x=\int_{0}^{\infty} f \phi_{n}^{(\alpha, m)} d x=0, \quad[n=0,1,2, \ldots] .
$$

$\left\{\psi_{n}^{(a+2 m)}\right\}$ being complete with respect to $L^{2}(0, \infty)$ because $\mathscr{R}(\alpha)+2 m>-1$, $F(x)$ is a nul-function. On the other hand from (50) it is seen that $F(x)$ is absolutely continuous and $m$ times differentiable in any finite interval $0<x_{1} \leqq x \leqq x_{2}$. Hence $F(x)$ is identically zero. Hence $f(x)$ is a nul-function.

Thus the completeness of $\left\{\chi_{n}^{(\alpha, m)}, \phi_{n}^{(a, m)}\right\}$ in $L^{2}(0, \infty)$ is proved. From a well-known general theorem it then follows that this biorthogonal sequence is also closed in $L^{2}(0, \infty)$. Furthermore from a general theorem of $\operatorname{Kober}^{1}$ it is seen that $\left\{\chi_{2 n}^{(a, m)}, \phi_{2 n}^{(a, m)}\right\}$ and $\left\{\chi_{2 n+1}^{(a, m)}, \phi_{2 n+1}^{(a, m)}\right\}$ are closed in the respective function-spaces consisting of functions belonging to $L^{2}(0, \infty)$ and being self or skew reciprocal respectively in the cut Hankel transformation.

Thus the remark at the end of the preceding section can also be expressed by saying that every function which belongs to $L^{2}(0, \infty)$ and is self or skew reciprocal in the cut Hankel transformation $\mathscr{H}_{a, m}$ can be respectively approximated to by finite linear combinations of the $\chi_{2 n}^{(a, m)}(x)$ only or of the $\chi_{2 n+1}^{(a, m)}(x)$ only.

For the sake of simplicity I have restricted myself throughout the paper to the class $L^{2}(0, \infty)$. It is quite clear that corresponding theorems hold for classes $L^{p}(0, \infty)$, the restriction $-1<\mathscr{R}(a)+2 m<1$ being then replaced by $-2 / p<\mathcal{R}(a)+2 m<2-2 / p$.

Some remarks on Hankel and general transforms.

12. The work of the preceding ten sections depends entirely on special features of the Hankel transformation (the general theorem of

1 H. Kober, Ann. of Math., 40 (1939), 549-59, Satz IV. 
section 3 for instance on the formulae for the derivatives of Bessel functions) and on the knowledge of an explicit expression for the reciprocal functions. In what follows some remarks are made on alternative proofs of some of the results of the preceding sections. These alternative proofs are independent of the special features of the Hankel transform and apply likewise to a class of general transforms, namely, roughly speaking, to general transforms possessing a reciprocal function which is an analytic function of the complex variable $x+i t$ and regular for all positive finite values of $x,(t=0)$. Applied to Hankel transforms, no new results will be obtained, only old ones regained. Therefore I do not give rigorous proofs in this chapter, but formal sketches only.

First a few comments may be made on the generating function and the role it plays.

From (22) and (39) in connection with (17), (25) and (33), (40) respectively we obtain the generating functions of the sequences $\left\{\chi_{n}^{(a, m)}\right\}$ and $\left\{\phi_{n}^{(a, m)}\right\}$ :

$$
\begin{aligned}
& \sum_{n=0}^{\infty} u^{n} \chi_{n}^{(a, m)}(x)=(1+u)\left(1-u^{2}\right)^{-\frac{1}{2} a-m-1} \chi_{0}^{(a, m)}\left(\frac{1+u}{1-u} x\right),(|u|<1) \\
& \sum_{n=0}^{\infty} u^{n} \phi_{n}^{(\alpha, m)}(x)=(1+u)\left(1-u^{2}\right)^{-\frac{1}{d} a-m-1} \phi_{0}^{(a, m)}\left(\frac{1+u}{1-u} x\right),(|u|<1) .
\end{aligned}
$$

Hence in both cases the generating function is, except for a factor depending only on $u$, the "simplest" reciprocal function of the sequence, of argument $(1+u) x /(1+u)$. This is by no means a special feature of the Hankel transform.

Suppose a general transform is given which for the present purely formal investigations may be taken in the non-integrated form

$$
g(x)=\int_{0}^{\infty} K(x y) f(y) d y
$$

Suppose furthermore that a self-reciprocal function of the kernel $K(x y), \phi(x)$ say, is known which is an analytic function of the complex variable $x+i t$, regular for $0<x<\infty, t=0$. This function is a solution of the integral equation

$$
\phi(x)=\int_{0}^{\infty} K(x y) \phi(y) d y .
$$

Replacing $x$ and $y$ by $(1+u) x /(1-u)$ and $(1-u) y /(1+u)$ respectively, $-1<u<1$, this integral equation transforms into

$$
(1+u) \phi\left(\frac{1+u}{1-u} x\right)=\int_{0}^{\infty} K(x y)(1-u) \phi\left(\frac{1-u}{1+u} y\right) d y
$$


Now if $a\left(u^{2}\right)$ is a power-series in $u^{2}$ the first term of which is unity , and functions $\phi_{n}(x)$ are defined by the generating function

$$
\sum_{n=0}^{\infty} u^{n} \phi_{n}(x)=(1+u) a\left(u^{2}\right) \phi\left(\frac{1+u}{1-u} x\right),
$$

then $\phi_{n}(x)$ is an eigen-function of the kernel $K(x y)$ belonging to the eigen-value $(-)^{n}$. This is easily seen, for, by multiplying (54) by $a\left(u^{2}\right)$ and expanding both sides, (54) transforms into

$$
\sum_{n=0}^{\infty} u^{n} \phi_{n}(x)=\sum_{n=0}^{\infty}(-u)^{n} \int_{0}^{\infty} K(x y) \phi_{n}(y) d y
$$

This being true for every $u$ in the circle of convergence of (55), coefficients of equal powers of $u$ on both sides must be equal, yielding

$$
\phi_{n}(x)=(-)^{n} \int_{0}^{\infty} K(x y) \phi_{n}(y) d y, \quad(n=0,1,2, \ldots)
$$

the reciprocal property of the $\phi_{n}$. If $a\left(u^{2}\right)$ is a power-series and $a(0)=1$ then $\phi_{0}(x)=\phi(x)$. If $a\left(u^{2}\right)$ contains also negative powers of $u$, then $\phi(x)$ does not necessarily occur as an element of the sequence $\left\{\phi_{n}\right\}$.

13. The generating function may be used in investigating the orthogonality or biorthogonality as well as completeness and closedness of the system of reciprocal functions generated.

(i) Orthogonality. If $\left\{\phi_{n}\right\}$ is an orthogonal system, that is, if

$$
\int_{0}^{\infty} \phi_{m} \phi_{n} d x=c_{n} \delta_{m n},
$$

then

$$
(1+u)(1+v) a\left(u^{2}\right) a\left(v^{2}\right) \int_{0}^{\infty} \phi\left(\frac{1+u}{1-u} x\right) \phi\left(\frac{1+v}{1-v} x\right) d x=\sum_{u=0}^{\infty} u^{n} v^{n} c_{n}=F(u v) .
$$

Hence from a known self-reciprocal function $\phi(x)$ an orthogonal sequence of self and skew reciprocal functions can be generated if and only if the integral

$$
\int_{0}^{\infty} \phi\left(\frac{1+u}{1-u} x\right) \phi\left(\frac{1+v}{1-v} x\right) d x
$$

is equal to $(1+u)^{-1}(1+v)^{-1}$ multiplied by a function of $u^{2}$ only multiplied by the same function of $v^{2}$ multiplied by any function of

1 Dr Kober, in a letter, remarks that, of course, $a\left(u^{2}\right)$ may be a Laurent series as well. In this case the summation has to run from $-\infty$ to $+\infty$. 
$u v$ only. If this is the case, the corresponding power (or Laurent) series $a\left(u^{2}\right)$ (except for a factor $C u^{2 n}$ in the case of a Laurent series) and also the $c_{n}$ are uniquely determined except, possibly, for constant factors, common to all $c_{n}$.

(ii) Biorthogonality. Suppose that there are two known selfreciprocal functions $\phi(x)$ and $\chi(x)$. If and only if the integral

is of form

$$
\int_{0}^{\infty} \phi\left(\frac{1+u}{1-u} x\right) \times\left(\frac{1+v}{1-v} x\right) d x
$$

$$
\left[(1+u)(1+v) a\left(u^{2}\right) b\left(v^{2}\right)\right]^{-1} F(u v),
$$

where $a\left(u^{2}\right), b\left(v^{2}\right), F(u v)$ are functions of their respective arguments only, from $\phi(x)$ and $\chi(x)$ a biorthogonal system $\{\phi, \chi\}$ can be generated by

and

$$
(1+u) a\left(u^{2}\right) \phi\left(\frac{1+u}{1-u} x\right)=\Sigma u^{n} \phi_{n}(x)
$$

for which

$$
(1+v) b\left(v^{2}\right) \times\left(\frac{1+v}{1-v} x\right)=\Sigma v^{n} \chi_{n}(x),
$$

$$
\int_{0}^{\infty} \phi_{m} \chi_{n} d x=c_{n} \delta_{m n}
$$

$c_{n}$ being the coefficient of $(u v)^{n}$ in the power-series expansion of $F(u v)$.

When this test is applied to the functions

$$
\phi_{0}^{(a, m)}(x)=(-)^{m} x^{\frac{1}{a} a} E_{m}(x)
$$

and

$$
\chi_{0}^{(a, m)}(x)=x^{1 a+m} e^{-x} L_{m}^{(a+m)}(x),
$$

it shows that $\left\{\chi_{n}^{(a, m)}, \phi_{n}^{(a, m)}\right\}$ is the only biorthogonal system which can be generated from $\chi_{0}^{(a, m)}(x)$ and $\phi_{0}^{(a, m)}(x)$ in a way like (55).

(iii) Completeness and closedness. The system $\left\{\phi_{n}\right\}$ generated by $(55)$ is said to be complete with respect to a certain space of functions if there is, except for nul-elements, no function $f(x)$ belonging to this space and satisfying every equation

$$
\int_{0}^{\infty} f \phi_{n} d x=0
$$

Multiplying (58) by $u^{n}$ and summing up with respect to $n$, it is seen that, at least formally, (58) and

$$
\int_{0}^{\infty} \phi\left(\frac{1+u}{1-u} x\right) f(x) d x=0
$$


are equivalent. Hence the completeness of $\left\{\phi_{n}\right\}$ depends on solutions of the homogeneous integral equation of the first kind (59) which belong to the class of functions with respect to which the completeness has to be proved. If there is any solution then clearly $\left\{\phi_{n}\right\}$ is not complete. If there is no solution then $\left\{\phi_{n}\right\}$ is complete.

This can also be applied to our system $\left\{x_{n}^{(a, m)}, \phi_{n}^{(a, m)}\right\}$.

The same criterion may be expressed in different words. In the next section the integral operator $T_{s}$ will be introduced which isdefined by

$$
T_{8}\{f\}=s^{\frac{1}{2}} \int_{0}^{x} \phi(s x) f(x) d x .
$$

Then $\left\{\phi_{n}\right\}$ is complete if and only if $T_{s}$ is total ${ }^{1}$.

The closedness of the sequence $\left\{\phi_{n}\right\}$ in $L^{p}(0, \infty)$, say, can be inferred, due to well-known general theorems, from its being complete with respect to $L^{p^{\prime}}(0, \infty)$ where $1 / p+1 / p^{\prime}=1$.

Thus it is seen that the power-series $a\left(u^{2}\right)$ is of no influence on the completeness or closedness of the generated sequence $\left\{\phi_{n}\right\}$. This means that all sequences generated from the same reciprocal function $\phi(x)$ by (55) with different power-series $a\left(u^{2}\right)$, though different as regards orthogonality or biorthogonality, whichever may be the case, are equivalent regarding completeness and closedness. This is quite clear, for the functions of the one set can be expressed as finite linear combinations of the functions of the other set.

Here also the relations between Kober's sequence of eigenfunctions of $\delta f_{a, m}$ and the sequence $\left\{\phi_{n}^{(a, m)}\right\}$ of this paper may be mentioned. Kober's set of reciprocal functions of $\forall t_{a, m}$ is defined by the generating function ${ }^{2}$

$$
\begin{aligned}
& \sum_{n=0}^{\infty} u^{n} \psi_{n, \mathrm{j} m}^{(a)}(2 x)=(1-u)^{-a-1}(2 x)^{\frac{1}{a} a} E_{m}\left(\frac{1+u}{1-u} x\right) \\
& \quad=(-)^{m} 2^{\frac{1}{1} a}(1+u)\left(1-u^{2}\right)^{-\frac{1}{2} a-1} \phi_{0}^{(a, m)}\left(\frac{1+u}{1-u} x\right),(|u|<1) .
\end{aligned}
$$

Thus from what has been said at the end of (ii) it is clear that no sequence of reciprocal functions can be generated from $\chi_{0}^{(a, m)}(x)$ which completes $\left\{\psi_{n, m}^{(a)}\right\}$ to a biorthogonal system. Moreover from com-

${ }^{2}$ For the definition of total operators see S. Batnach, Théorie des opérations linéaires (Warszawa, 1932), p. 42.

2 H. Kober, Proc. Edinburgh Math. Soc. (2), 6 (1940), 135-146 (3.5). 
parison of (61) and (53) Kober's functions may be expressed easily in terms of those introduced here and vice versa. This comparison yields

$$
\psi_{n, m}^{(a)}(2 x)=(-)^{m} 2^{\frac{1}{a} a} \Sigma\left(\begin{array}{c}
m \\
r
\end{array}\right)(-)^{r} \phi_{n-2 r}^{(a, m)}(x),
$$

the summation being extended over all non-negative integers $r$ not larger than $m$ and not larger than $\frac{1}{2} n$.

Obviously the remarks put forward in this section have no bearing at all on the question of whether the functions dealt with are reciprocal functions of certain functional transformations. Mutatis mutandis they apply to any sequence of functions generated by a power-series.

Plainly the Mellin and Laplace transforms as well as any other linear functional operations can be obtained from the generating functions.

14. A few more remarks on the operation $T_{8}$ may be added. Let us begin with the Hankel transform.

It is well known that the way of dealing with the Hankel transform is, to "translate" it by some functional transformation into a simpler relation. There are two essentially different ways of doing this, namely, by the Mellin transform or by the Laplace transform.

The application of Mellin's transform to (1) is so well known that I need not say much about it. There are two functions $F(t)$ and $G(t)$ corresponding in a certain way to $f(x)$ and $g(x)$ respectively (they are also dependent on $a$ ) so that the relation (1) between $f(x)$ and $g(x)$ corresponds to the simple relation

$$
F(t)=G(-t)
$$

between $F$ and $G$. Hence the functional transformation " $\mathscr{F}_{a}$ in the space of the functions $f$ corresponds in the space of the "images" $F$ to the reflection in the point $t=0$ of the (complex) $t$-plane. From this all the fundamental properties of the Hankel transform immediately follow. Obviously $\mathcal{H}_{\alpha}$ is identical with its own inversion (so is the reflection), its only eigenvalues are \pm 1 , and the eigen-functions $\phi(x)$ are characterised by the relation

$$
\Phi(t)= \pm \Phi(-t)
$$

between their images.

Fortunately the same method also applies to general transforms. 
Watson ${ }^{1}$ himself in his original work used it, although only heuristically, and Miss Busbridge ${ }^{2}$ proved the transform theory in this way. Translation by means of Mellin transform is also the basis of Kober's. theory of general transforms $\mathrm{m}^{3}$ and hence of his theory of the cut Hankel transform too*.

It is quite natural that translation by means of the Mellin transform, being universally applicable to all general transforms, thekernel of which depends on the product of $x$ and $y$ only, should be esteemed as the way of understanding Hankel transforms. It is. comparatively little known that Tricomi ${ }^{5}$ gave another theory of Hankel transform which is surely not less beautiful and not lesssimple-as regards the reciprocal functions perhaps even simpler ${ }^{6}$.

Tricomi uses the Laplace transform

$$
\mathcal{L}_{s}\{f\} \equiv \int_{0}^{\infty} e^{-s x} f(x) d x
$$

and attaches to the functions $f(x), g(x)$ as "images"

$$
F(s)=s^{\frac{1}{3} a+\frac{1}{2}} \mathcal{L}_{8}\left\{x^{\frac{1}{2} a} f(x)\right\}
$$

and

$$
G(s)=s^{\frac{1}{2} a+\frac{1}{2}} \mathcal{L}_{8}\left\{x^{\frac{1}{2} a} g(x)\right\}
$$

respectively. Then the connection (1) between $f$ and $g$ corresponds to the relation $G(s)=F(1 / s)$ between their images. Hence the Hankel transform in the space of the functions $f, g$ corresponds, in the space of the images $F$ and $G$, simply to the inversion $s^{\prime}=1 / \mathrm{s}$ of the complex variable $s$. From this again all the characteristic

${ }^{1}$ G. N. Watson, Proc. London Math. Soc. (2), 35 (1933), 156-99.

2 I. W. Busbridge, Journal London Math. Soc, 9 (1934), $179-87$.

${ }^{3}$ H. Kober, Quart. J. of Math. (Oxford Series), 8 (1937), 172-85.

4 H. Kober, Ibidem, 186-99.

${ }^{5}$ F. Tricomi, Rend. dei Lincei (6), 22 (1935), 564-71 and 572-6. Atti Torino, 71 (1936), 285-91.

${ }^{6}$ Tricomi's method has been used in connection with the operational calculus. B. van der Pol and K. F. Niessen, Phil. Mag. (7), 13 (1932), 537-75, eventually established Tricomi's theorem as a "rule" in the particular cases $a=0,1$ before Tricomi dealt with the general case. K. F. Niessen, Phil. Mag. (7), 20 (1935), 977-97, formulated the general rule for $a=0,1,2, \ldots \ldots$ independent from and at the same time as Tricomi. This rule has been frequently used by several authors for evaluation of Hankel transforms. 
properties of Hankel transform immediately follow. Reciprocal functions, in particular, are determined by the equation

$$
\Phi(s)= \pm \Phi(1 / s)
$$

which their images must satisfy.

In practice Tricomi's translation works more simply with reciprocal functions than translation by means of the Mellin transform. The image in the case of the Mellin transform (it corresponds to $\Gamma\left(\frac{a+1-i t}{2}\right) \Phi_{n}^{(a, 0)}(t)$ in the notation of equation (44)) is a terminating hypergeometric series, and it is only from the transformation theory of hypergeometric series that (45) follows. The image of $\psi_{n}^{(a)} \equiv \phi_{n}^{(a, 0)}$ in the sense of $(63)$, as is seen from (46), is however

$$
s^{\frac{1}{a} a+\frac{1}{2}} \mathcal{s}_{s}\left\{x^{\frac{1}{2} a} \psi_{n}^{(a)}(x)\right\}=\frac{\Gamma(a+n+1)}{n !} \frac{s^{j a+1}(s-1)^{n}}{(s+1)^{a+n+1}},
$$

and this is not only a simpler expression than (44) (with $m=0$ ) but also exhibits immediately that (64) is fulfilled.

Unfortunately the Laplace transform does not work in the case of general transforms of the form

$$
g(x)=\int_{0}^{\infty} K(x y) f(y) d y .
$$

I have always wondered why of the two transforms, equally useful in the case of the Hankel transform, equally general and useful in analysis, one should be applicable also to general transforms and the other not.

It is quite obvious why the Mellin transform applies to the general case. (65) as well as (1) is of the form of a resultant as appearing in connection with the Mellin transform, and it is quite natural that the Mellin transform should "algebraicise" the relation between $f$ and g. But why then does the Laplace transform work with (1), although this is by no means the type of resultant which occurs in connection with the Laplace transform? The answer is: It is really not the Laplace transform which has been applied by Tricomi, but the transform

$$
T_{s}\{f\}=s^{1} \int_{0}^{\infty} \psi_{0}^{(\alpha)}(s x) f(x) d x,
$$

and it is only because (1) happens to have a self-reciprocal function $\psi_{0}^{(a)}(x)=e^{-x} x^{\frac{1}{2} a}$ that $T_{s}\{f\}$ in this case reduces, except for a factor, to the Laplace transform of $x^{\frac{1}{2} a} f(x)$. 
Now it is seen how the operation $T_{\delta}$ is "adjusted" to the special nature of the Hankel transform-and how it should be generalised so as to suit (65).

If $\phi(x)$ is any self-reciprocal function of (65) and $T_{8}$ is defined. by (60) then, for a pair of functions satisfying (65),

$$
T_{8}\{g\}=T_{1 / 8}\{f\} .
$$

Self or skew reciprocal functions $f(x)$ are characterised by the relations.

$$
T_{s}\{f\}= \pm T_{1 / 8}\{f\}
$$

respectively, everything in exact analogy to Tricomi's theory of the. Hankel transform ${ }^{1}$.

Obviously a skew reciprocal function can be used as kernel of $T_{s}$ as well, there being only a difference of sign in (66).

15. I do not mean to suggest that the operation $T_{8}$ should be made the basis of the theory of general transforms. The choice from the point of view of the general theory is not difficult indeed, seeing that the Mellin transform applies universally to all kernels $K(x y)$ whereas the operation $T_{s}$ depends on the kernel. Besides, the operation $T_{s}$ cannot be made to work unless one has knowledge of the existence and at least some properties of a reciprocal function. But, although not of very much use from the point of view of the general theory, $T_{s}$ may work quite well with certain special transforms.

Now the not very convincing argument of section 7 for "cutting" the Laplace transform in order to apply it to the reciprocal function of the cut Hankel transform can be replaced by the right explanation. All that happened was that $\psi_{0}^{(a)}(s x)$, which applies to the ordinary Hankel transform, was replaced by $\phi_{u}^{(a, m)}(s x)$ which plays the corresponding role with the cut Hankel transform. (32) should be written

$$
s^{\frac{1}{3}} \int_{0}^{\infty} \phi_{0}^{(a, m)}(s x) \chi_{n}^{(a, m)}(x) d x=\frac{\Gamma(\alpha+2 m+n+1)}{(-)^{m} m ! n !} \frac{s^{\frac{1}{a} a+m+\frac{1}{2}}(s-1)^{n}}{(s+1)^{a+2 m+n+1}} .
$$

Similarly the modification of the Laplace transform used in section 9 explains itself when (46) is written in the form

$$
s^{1} \int_{0}^{\infty} \chi_{0}^{(a, m)}(s x) \phi_{n}^{(a, m)}(x) d x=\frac{\Gamma(a+2 m+n+1)}{(-)^{m} m ! n !} \frac{s^{\frac{1}{a+m+b}}(s-1)^{n}}{(s+1)^{a+2 m+n+1}} .
$$

The right hand sides of both (68) and (69) exhibit the property (67)

\footnotetext{
${ }^{1}$ Of. also $\$ 9.3$ of Titchmarsh's Fourier integrals.
} 
and so these relations are a new proof of the reciprocal property of the $\left\{\chi_{n}^{(a, m)}\right\}$ and $\left\{\phi_{n}^{(a, m)}\right\}$.

A few more comments on (68) and (69) are perhaps useful. Of course, any reciprocal function could be used as kernel of the operation $T_{s}$ in this case. $\chi_{0}^{(a, m)}$ and $\phi_{0}^{(a, m)}$ are the simplest ones known. 'The advantage of using these arises from the fact that they are the generating functions of the sequences $\left\{\chi_{n}^{(a, m)}\right\}$ and $\left\{\phi_{n}^{(a, m)}\right\}$ respectively. Applying $\chi_{0}^{(a, m)}$ to $\left\{\phi_{n}^{(a, m)}\right\}$ and vice versa gives simple results because of the biorthogonality of $\left\{\chi_{n}^{(a, m)}, \phi_{n}^{(a, m)}\right\}$.

Suppose we have two self-reciprocal functions $\phi(x)$ and $\chi(x)$ of (65), and that, by

and

$$
\sum u^{n} \phi_{n}(x)=(1+u) a\left(u^{2}\right) \phi\left(\frac{1+u}{1-u} x\right)
$$

$$
\Sigma u^{n} \chi_{n}(x)=(1+u) b\left(u^{2}\right) \chi\left(\frac{1+u}{1-u} x\right),
$$

a biorthogonal system $\left\{\phi_{n}, \chi_{n}\right\}$ is generated such that

'Then the operation

$$
\int_{0}^{\infty} \phi_{m} \chi_{n} d x=c_{n} \delta_{m n}
$$

$$
T_{s}\{f\} \equiv s^{\frac{1}{2}} \int_{0}^{\infty} \phi(s x) f(x) d x
$$

gives most simple results when applied to $\left\{\chi_{n}\right\}$, and the operation

$$
T_{s}^{\prime}\{f\} \equiv s^{\xi} \int_{0}^{\infty} \chi(s x) f(x) d x
$$

applies to $\left\{\phi_{n}\right\}$. For, from the generating function,

and hence

$$
s^{\frac{1}{2}} \phi(s x)=\frac{s+1}{2 \sqrt{ } s} \Sigma\left(\frac{s-1}{s+1}\right)^{m} \phi_{m}(x) / a\left[\left(\frac{s-1}{s+1}\right)^{2}\right],
$$

$$
\begin{aligned}
s^{\frac{1}{2}} \int_{0}^{\infty} \phi(s x) \chi_{n}(x) d x & =\frac{s+1}{2 \sqrt{ } s} \Sigma\left(\frac{s-1}{s+1}\right)^{m} \int_{0}^{\infty} \phi_{m} \chi_{n} d x / a\left[\left(\frac{s-1}{s+1}\right)^{2}\right] \\
& =\frac{s+1}{2 \sqrt{ } s}\left(\frac{s-1}{s+1}\right)^{n} c_{n} / a\left[\left(\frac{s-1}{s+1}\right)^{2}\right]
\end{aligned}
$$

and similarly

$$
s^{\frac{1}{2}} \int_{0}^{\infty} \chi(s x) \phi_{n}(x) d x=\frac{s+1}{2 \sqrt{ } s}\left(\frac{s-1}{s+1}\right)^{n} c_{n} / b\left[\left(\frac{s-1}{s+1}\right)^{2}\right] .
$$


This is in exact agreement with (68) and (69). In the case of thebiorthogonal system $\left\{\chi_{n}^{(a, m)}, \phi_{n}^{(\alpha, m)}\right\}$ moreover, $a\left(u^{2}\right)$ and $b\left(u^{2}\right)$ are identical. Hence the identity of the right hand sides of (68) and (69).

Another biorthogonal set.

16. I conclude by introducing another biorthogonal set. Although the sequence of biorthogonal functions which will be introduced below has no connection at all with the cut Hankel transform, yet it. is an extension of Laguerre's orthogonal set

$$
\left\{e^{-\jmath x} x^{! x} L_{n}^{(a)}(x)\right\}, \quad(n=0,1,2, \ldots), \quad[-\mathcal{R}(a)>-1]
$$

to values of $\mathcal{C}(a)$ which are less than -1 . This set is in many ways simpler than the system $\left\{\chi_{n}^{(a, m)}, \phi_{n}^{(a, m)}\right\}$. Like Laguerre's set, it consists only of functions which, except for a common factor, are polynomials, these polynomials being (i) Laguerre polynomials (7), and (ii) "cut" Laguerre polynomials

$$
\begin{aligned}
& L_{n, l}^{(a)}(x)=\sum_{r=l}^{n}\left(\begin{array}{l}
n+a \\
n-r
\end{array}\right) \frac{(-x)^{r}}{r !} \\
& =\left(\begin{array}{l}
n+a \\
n-l
\end{array}\right) \frac{(-x)^{l}}{l !}{ }_{2} F_{2}(l-n, 1 ; l+a+1, l+1 ; x), \quad(l \leqq n)_{r}
\end{aligned}
$$

which are obtained by omitting the first $l$ terms in $L_{n}^{(a)}(x)$.

The biorthogonal set in question, $\left\{\phi_{n}, \psi_{n}\right\}$, is defined by

$$
\phi_{n}(x)=e^{-\frac{1}{2} x} x^{\frac{1}{(a+l)}} L_{n+l}^{(a)}(x), \quad(n=0,1,2, \ldots),
$$

and

$$
\psi_{n}(x)=e^{-\frac{1}{2} x} x^{\frac{1}{2}(a-l)} L_{n+l, l}^{(a)}(x), \quad(n=0,1,2, \ldots) .
$$

Obviously both $\phi_{n}$ and $\psi_{n}$ belong to $L^{2}(0, \infty)$ if only $\mathscr{R}(a)>-l-1$. This will be assumed throughout the rest of the paper.

In the paper referred to at the end of section 10, I gave a proof of the biorthogonality of $\left\{\phi_{n}, \psi_{n}\right\}$ very similar to the proof of the biorthogonality of $\left\{\chi_{n}^{(a, m)}, \phi_{n}^{(a, m)}\right\}$ given in this paper. It is also easy to prove the completeness and closedness of $\left\{\phi_{n}, \psi_{n}\right\}$ in the same way as that of $\left\{\chi_{n}^{(a, m)}, \phi_{n}^{(a, m)}\right\}$. Here I shall give alternative proofs using the methods of section 13 .

First let us find the generating functions of $\left\{\phi_{n}\right\}$ and $\left\{\psi_{n}\right\}$. From (8)

$$
L_{n+l}^{(a)}(x)=\frac{e^{x} x^{-a}}{(n+l) !} \frac{d^{n+l}\left(e^{-x} x^{x+n+l}\right)}{d x^{n+l}}=\frac{n !}{(n+l) !} e^{x} x^{-a} \frac{d^{l}}{d x^{l}}\left\{e^{-x} x^{x+l} L_{n}^{(a+l)}(x)\right\},
$$


and hence, from (6)

$$
\sum_{n=0}^{\infty} \frac{(n+l) !}{n !} u^{n} L_{n+l}^{(a)}(x)=e^{x} x^{-a}(1-u)^{-a-l-1} \frac{d^{l}}{d x^{l}}\left(e^{-x_{i}^{\prime}(1-u)} x^{a+l}\right),
$$

or, using (8) again,

$$
\sum_{n=0}^{\infty} \frac{(n+l) !}{n ! l !} u^{n} L_{n+l}^{(a)}(x)=(1-u)^{-a-l-1} e^{-u x /(1-u)} L_{l}^{(a)}\left(\frac{x}{1-u}\right),(|u|<1) .
$$

Similarly

and we obtain

$$
L_{n+l, l}^{(a)}(x)=\underset{(l-1) !}{(-)^{l}} \int_{0}^{\infty}(x-y)^{l-1} L_{n}^{(a+l)}(y) d y,
$$

$$
\sum_{n=0}^{\infty} u^{n} L_{n+l, l}^{(a)}(x)=u^{-l}(1-u)^{-a-1} E_{l}\left(\frac{u x}{1-u}\right), \quad(|u|<1) .
$$

Thus the generating function of $\left\{\phi_{n}\right\}$ is

(78) $\sum_{n=0}^{\infty} \frac{(n+l) !}{n ! l !} u^{n} \phi_{n}(x)=(1-u)^{-a-l-1} x^{\underline{3}(a+l)} e^{-\frac{1}{i} x(1+u) /(1-u)} L_{l}^{(a)}\left(\frac{x}{1-u}\right),(|u|<1)$, and that of $\left\{\psi_{n}\right\}$ is

(79) $\sum_{n=0}^{\infty} u^{n} \psi_{n}(x)=u^{-l}(1-u)^{-a-1} x^{1(a-l)} e^{-\frac{b}{2} x} E_{l}\left(\begin{array}{c}u x \\ 1-u\end{array}\right), \quad(|u|<1)$.

In order to establish the biorthogonality of $\left\{\phi_{n}, \psi_{n}\right\}$, the integral

$$
\begin{aligned}
& \int_{0}^{\infty} \sum_{n=0}^{\infty} \frac{(m+l) !}{m ! l !} u^{m} \phi_{m} \sum_{n=0}^{\infty} v^{n} \psi_{n} d x \\
& =(1-u)^{-a-l-1} v^{-l}(1-v)^{-a-1} \int_{0}^{\infty} e^{-x /(1-u)} x^{\alpha} L_{l}^{(\alpha)}\left(\frac{x}{1-u}\right) E_{l}\left(\frac{v x}{1-v}\right) d x
\end{aligned}
$$

has to be evaluated. By (8) this is

$$
\frac{1}{l !}(1-u)^{-a-l-1} v^{-l}(1-v)^{-a-1} \int_{0}^{\infty} \frac{d^{l}}{d x^{l}}\left(e^{-x / 1-u)} x^{a+l}\right) E_{l}\left(\frac{v x}{1-v}\right) d x .
$$

Integrating $l$ times by parts this becomes

$$
\begin{aligned}
\frac{1}{l !}[(1-u) & (1-v)]^{-a-l-1} \int_{0}^{\infty} e^{-x \mid 1 /(1-u)+1 /(1-v) l} x^{a+l} d x \\
= & \frac{\Gamma(a+l+1)}{l !}(1-u v)^{-a-l-1}=\sum_{n=0}^{\infty} \frac{\Gamma(a+l+n+1)}{n !} \frac{l+n}{l !} v^{n} .
\end{aligned}
$$

\section{Hence}

$$
\int_{0}^{\infty} \phi_{m} \psi_{n} d x=\frac{\Gamma(a+l+n+1)}{(n+l) !} \delta_{m n}
$$


According to section 13 (iii), the completeness of $\left\{\phi_{n}, \psi_{n}\right\}$ depends on solutions of the integral equation of the first kind

$$
\int_{0}^{\infty} e^{-\frac{1}{2} x} x^{\frac{1}{(a-l)}} E_{l}\left(\frac{u x}{1-u}\right) f(x) d x=0 .
$$

The proof that this equation has no solution in $L^{2}(0, \infty)$ is very similar to the proof of the completeness of $\left\{\chi_{n}^{(a, m)}, \phi_{n}^{(a, m)}\right\}$ in section 11 . The function $F(x)$ has to be defined here by

$$
F(x)=x^{-\frac{1}{(2}(a+l)} \cdot \frac{1}{(l-1) !} \int_{x}^{\infty}(y-x)^{l-1} e^{-\frac{1}{2} y} y^{\frac{1}{2}(a-l)} f(y) d y .
$$

If $f(x)$ belongs to $L^{2}(0, \infty)$, clearly $e^{-\frac{1}{2} x} f(x)$ and also $F(x)$ belong to $L(0, \infty)$. Integrating (81) $l$ times by parts, we have, since

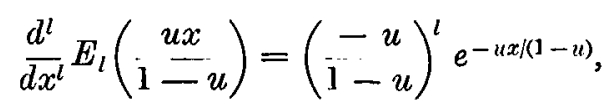

instead of (81),

$$
\int_{0}^{\infty} e^{-u x /(1-u)} F(x) d x=0 .
$$

This being true identically in $u$ (for $0<u<1$, say), it follows from Lerch's theorem that $F(x)=0$ almost everywhere. From this $f(x)=0$ almost everywhere, and hence the completeness of the sequence $\left\{\phi_{n}, \psi_{n}\right\}$ can be inferred.

Mathematical Institute,

The University, Edinburgh. 